\title{
Reduction of discrete interval system using clustering of poles with Padé approximation: a computer-aided approach
}

\author{
V.P. Singh ${ }^{1 *}$, D. Chandra ${ }^{2}$ \\ 1,2 Department of Electrical Engineering, M. N. National Institute of Technology, Allahabad-211004, INDIA \\ *Corresponding Author: e-mail: vinaymnnit@gmail.com, Tel +91-9452793089
}

\begin{abstract}
This paper presents a method for the order reduction of discrete interval systems using pole-clustering method along with Padé approximation technique. For a given discrete high-order interval system (HOIS), the denominator of the reduced-order interval model (ROIM) is obtained by pole-clustering while the numerator is obtained by minimizing errors between first $r$ time moments of the HOIS to those of its ROIM where $r$ is the order of ROIM. Inverse distance measure (IDM) criterion is used for clustering of poles of HOIS whereas the time moments of HOIS (or ROIM) are obtained without inverting the denominator of transfer function and presented in generalized form. The error function which is weighted squared sum of errors between first $r$ time moments of HOIS and those of its ROIM is carried out by the algorithm due to Luus-Jaakola. The distinctive feature of this method is that the numerator coefficients are treated free in the process of minimization. A numerical example is given to illustrate the procedure.
\end{abstract}

Keywords: Discrete interval system; Inverse distance measure; Kharitonov polynomials; Model reduction; Padé approximation; Pole-clustering method.

\section{Introduction}

Due to several advantages e.g. reduced computational effort in simulation, simplified understanding of system, simpler control laws etc., model reduction has been ample area of research. Several methods have been proposed for reduction of continuous-time and discrete-time systems (Shamash, 1974; Aoki, 1968; Glover, 1984; Sinha and Kuszta, 1983; Huttan and Friedland, 1975; Shamash, 1975; Rao el al., 1978; Singh el al., 2004; Singh et al., 2004; Prajapati et al., 2007). Among them, Padé approximation method (Shamash, 1974) has found to be very useful in theoretical physics research (Baker and Graves-Morris, 1981; Baker, 1975) due to being computationally simple but the reduced-order model obtained using Padé approximation method often leads to be unstable even though the high-order system is stable. To overcome limitaion, many improvements have been proposed (Huttan and Friedland, 1975; Shamash, 1975; Rao el al., 1978; Singh el al., 2004; Singh et al., 2004; Prajapati et al., 2007) in literature.

The order reduction of HOISs has also attracted researchers since the pioneering work by Kharitonov (Kharitonov, 1978). Some methods have been presented for order reduction of HOISs (Bandyopadhyay et al., 1994; Dolgin and Zeheb, 2003; Bandyopadhyay et al., 1997; Sastry et al., 2000; Ismail and Bandyopadhyay, 1995; Ismail et al., 1997; Singh and Chandra, 2011). Among these, Routh-Padé approximation (Bandyopadhyay et al., 1994) has been presented for continuous HOISs in which the numerator is obtained by matching the time moments and the denominator is obtained by direct truncation of the Routh table. However, Dolgin and Zeheb (Dolgin and Zeheb, 2003) showed that a stable interval polynomial may provide an unstable interval polynomial if it is obtained by direct truncation of the Routh table as in (Bandyopadhyay et al., 1994).

In (Bandyopadhyay et al., 1997), a ROIM is proposed for model reduction of continuous HOISs using parameters. But this method becomes complex as it requires both and tables for obtaining ROIM. An improvement (Sastry et al., 2000) in which the numerator and denominator of ROIM both are obtained using parameters is suggested over (Bandyopadhyay et al., 1997). A 
method for reduction of discrete HOISs (Ismail et al., 1997) using Padé approximation and retention of dominant poles is proposed where Padé approximation is applied for obtaining the numerator and the denominator is obtained by retaining dominant poles of HOIS.

In this paper, a method, inspired by (Singh et al., 2004; Singh et al., 2004), for the model reduction of discrete HOISs is proposed where the time moments are obtained without inverting the denominator of transfer function of HOIS (or ROIM) in contrast to (Bandyopadhyay et al., 1994) in which the denominator is first inverted then time moments are determined. The denominator is obtained by pole-clustering technique (Vishwakarma and Prasad, 2008) and the numerator is obtained by matching the first $r$ time moments of HOIS to those of its ROIM. IDM method is used for clustering of poles of HOIS and the minimisation of error function, which is weighted squared sum of errors between first $r$ time moments of HOIS and those of its ROIM, is carried out by the algorithm due to Luss-Jaakola (Luus and Jaakola, 1973). The distinctive feature of proposed method is that the numerator coefficients are treated as free parameters in the process of minimization. The brief outline of this paper is as follows: section-2 covers problem formulation, section-3 contains algorithm due to Luss-Jaakola, section-4 contains numerical example and conclusion is given in section-5.

\section{Problem Formulation}

Consider a stable discrete HOIS given by the transfer function

$$
\begin{aligned}
& G(z)=\frac{\left[b_{0}^{-}, b_{0}^{+}\right]+\left[b_{1}^{-}, b_{1}^{+}\right] z+\cdots+\left[b_{n-1}^{-}, b_{n-1}^{+}\right] z^{n-1}}{\left[a_{0}^{-}, a_{0}^{+}\right]+\left[a_{1}^{-}, a_{1}^{+}\right] z+\cdots+\left[a_{n}^{-}, a_{n}^{+}\right] z^{n}} \\
& \quad=\left[t_{0}^{-}, t_{0}^{+}\right]+\left[t_{1}^{-}, t_{1}^{+}\right](z-1)+\cdots+\left[t_{n}^{-}, t_{n}^{+}\right](z-1)^{n}+\cdots
\end{aligned}
$$

(expansion around $z=1$ )

where $\left[b_{i}^{-}, b_{i}^{+}\right]$for $i=0,1, \cdots, n-1$ and $\left[a_{i}^{-}, a_{i}^{+}\right]$for $i=0,1, \cdots, n$ are the interval parameters. $b_{i}^{-}$and $b_{i}^{+}$are the lower and upper bounds of interval $\left[b_{i}^{-}, b_{i}^{+}\right]$, respectively and $a_{i}^{-}$and $a_{i}^{+}$are the lower and upper bounds of interval $\left[a_{i}^{-}, a_{i}^{+}\right]$, respectively. $\left[t_{i}^{-}, t_{i}^{+}\right]$for $i=0,1, \cdots$ are proportional to the time moments of HOIS. $t_{i}^{-}$and $t_{i}^{+}$are the lower and upper bounds of interval $\left[t_{i}^{-}, t_{i}^{+}\right]$, respectively.

Assuming $\left[b_{i}^{-}, b_{i}^{+}\right]=\boldsymbol{b}_{i}$ for $i=0,1, \cdots, n-1 ;\left[a_{i}^{-}, a_{i}^{+}\right]=\boldsymbol{a}_{i}$ for $i=0,1, \cdots, n$ and $\left[t_{i}^{-}, t_{i}^{+}\right]=\boldsymbol{t}_{i}$ for $i=0,1, \cdots$ (1)-(2) become (3)-(4), respectively.

$$
\begin{aligned}
& G(z)=\frac{\boldsymbol{b}_{0}+\boldsymbol{b}_{1} z+\cdots+\boldsymbol{b}_{n-1} z^{n-1}}{\boldsymbol{a}_{0}+\boldsymbol{a}_{1} z+\cdots+\boldsymbol{a}_{n} z^{n}} \\
& =\boldsymbol{t}_{0}+\boldsymbol{t}_{1}(z-1)+\cdots+\boldsymbol{t}_{n}(z-1)^{n}+\cdots
\end{aligned}
$$

Suppose, it is desired to obtain a stable $r$-th order $(r<n)$ model given as

$$
\begin{aligned}
& G_{r}(z)=\frac{\left[\hat{b}_{0}^{-}, \hat{b}_{0}^{+}\right]+\left[\hat{b}_{1}^{-}, \hat{b}_{1}^{+}\right] z+\cdots+\left[\hat{b}_{r-1}^{-}, \hat{b}_{r-1}^{+}\right] z^{r-1}}{\left[\hat{a}_{0}^{-}, \hat{a}_{0}^{+}\right]+\left[\hat{a}_{1}^{-}, \hat{a}_{1}^{+}\right] z+\cdots+\left[\hat{a}_{r}^{-}, \hat{a}_{r}^{+}\right] z^{r}} \\
& \quad=\left[\hat{t}_{0}^{-}, \hat{t}_{0}^{+}\right]+\left[\hat{t}_{1}^{-}, \hat{t}_{1}^{+}\right](z-1)+\cdots+\left[\hat{t}_{r}^{-}, \hat{t}_{r}^{+}\right](z-1)^{r}+\cdots
\end{aligned}
$$

(expansion around $z=1$ )

where $\left[\hat{b}_{i}^{-}, \hat{b}_{i}^{+}\right]$for $i=0,1, \cdots, r-1$ and $\left[\hat{a}_{i}^{-}, \hat{a}_{i}^{+}\right]$for $i=0,1, \cdots, r$ are the interval parameters. $\hat{b}_{i}^{-}$and $\hat{b}_{i}^{+}$are the lower and upper bounds of interval $\left[\hat{b}_{i}^{-}, \hat{b}_{i}^{+}\right]$, respectively and $\hat{a}_{i}^{-}$and $\hat{a}_{i}^{+}$are the lower and upper bounds of interval $\left[\hat{a}_{i}^{-}, \hat{a}_{i}^{+}\right]$, respectively. $\left[\hat{t}_{i}^{-}, \hat{t}_{i}^{+}\right]$for $i=0,1, \cdots$ are proportional to the time moments of the ROIM. $\hat{t}_{i}^{-}$and $\hat{t}_{i}^{+}$are the lower and upper bounds of interval $\left[\hat{t}_{i}^{-}, \hat{t}_{i}^{+}\right]$, respectively.

Assuming $\left[\hat{b}_{i}^{-}, \hat{b}_{i}^{+}\right]=\hat{\boldsymbol{b}}_{i}$ for $i=0,1, \cdots, r-1 ;\left[\hat{a}_{i}^{-}, \hat{a}_{i}^{+}\right]=\hat{\boldsymbol{a}}_{i}$ for $i=0,1, \cdots, r$ and $\left[\hat{t}_{i}^{-}, \hat{t}_{i}^{+}\right]=\hat{\boldsymbol{t}}_{i}$ for $i=0,1, \cdots$ (5)-(6) become (7)-(8), respectively.

$$
\begin{aligned}
& G_{r}(z)=\frac{\hat{\boldsymbol{b}}_{0}+\hat{\boldsymbol{b}}_{1} z+\cdots+\hat{\boldsymbol{b}}_{r-1} z^{r-1}}{\hat{\boldsymbol{a}}_{0}+\hat{\boldsymbol{a}}_{1} z+\cdots+\hat{\boldsymbol{a}}_{r} z^{r}} \\
& =\hat{\boldsymbol{t}}_{0}+\hat{\boldsymbol{t}}_{1}(z-1)+\cdots+\hat{\boldsymbol{t}}_{r}(z-1)^{r}+\cdots
\end{aligned}
$$

\subsection{Calculation of Poles of HOIS:}

Consider interval polynomial $F(z)$ given by 


$$
\left.\begin{array}{rl}
F(z) & =\left[f_{0}^{-}, f_{0}^{+}\right]+\left[f_{1}^{-}, f_{1}^{+}\right] z+\cdots+\left[f_{n-1}^{-}, f_{n-1}^{+}\right] z^{n-1}+z^{n} \\
& \equiv \boldsymbol{f}_{0}+\boldsymbol{f}_{1} z+\cdots+\boldsymbol{f}_{n-1} z^{n-1}+z^{n}
\end{array}\right\}
$$

The poles (Bandyopadhyay et al., 1997) of discrete interval polynomial $F(z)$ are calculated as:

Let $F$ be the interval matrix given as

$$
F=\left[\begin{array}{ccccc}
0 & 1 & 0 & \cdots & 0 \\
0 & 0 & 1 & \cdots & 0 \\
\vdots & \vdots & \vdots & \ddots & \vdots \\
0 & \vdots & \vdots & \cdots & 1 \\
-\boldsymbol{f}_{0} & -\boldsymbol{f}_{1} & -\boldsymbol{f}_{2} & \cdots & -\boldsymbol{f}_{n-1}
\end{array}\right]
$$

The interval matrix $F$ can be written as

$$
F=\left[F_{C}-\Delta F, F_{C}+\Delta F\right]
$$

where

$$
\left.\begin{array}{c}
f_{c i j}=\frac{1}{2}\left(f_{i j}^{-}+f_{i j}^{+}\right) \\
\Delta f_{c i j}=\frac{1}{2}\left(f_{i j}^{+}-f_{i j}^{-}\right)
\end{array}\right\} i, j=1,2, \cdots, n
$$

and $f_{i j}^{-}, f_{i j}^{+}$are the lower and upper bounds of $i j$ th element of $F$.

The real part $\lambda_{i}^{R}$ and imaginary part $\lambda_{i}^{I}$ of the ith eigenvalue $\lambda_{i}^{F}$ of $F$ are given as

$$
\left.\begin{array}{r}
\lambda_{i}^{R}(F)=\left[\lambda_{i}^{R}\left(F_{C}-\Delta F \circ U^{i}\right), \lambda_{i}^{R}\left(F_{C}+\Delta F \circ U^{i}\right)\right] \\
\lambda_{i}^{I}(F)=\left[\lambda_{i}^{I}\left(F_{C}-\Delta F \circ V^{i}\right), \lambda_{i}^{I}\left(F_{C}+\Delta F \circ V^{i}\right)\right] \\
i, j=1,2, \cdots, n
\end{array}\right\}
$$

where $\circ$ denotes component wise multiplication and elements of matrices $U^{i}$ and $V^{i}$ are given by

$$
\left.\begin{array}{l}
U_{k, j}^{i}=\operatorname{sgn}\left(n_{R k}^{i} m_{R j}^{i}+n_{I k}^{i} m_{I j}^{i}\right) \\
V_{k, j}^{i}=\operatorname{sgn}\left(n_{R k}^{i} m_{I j}^{i}-n_{I k}^{i} m_{R j}^{i}\right)
\end{array}\right\}
$$

where $m$ and $n$ are, respectively, the ith eigenvector and reciprocal eigenvector of $F_{C}$, with $R$ and $I$ denoting the real and imaginary parts, respectively.

\subsection{Calculation of Time Moments of HOIS and ROIM:}

Putting $z=p+1$ in (3), $G(z)$ becomes

$$
\begin{gathered}
G(p)=\frac{\boldsymbol{b}_{0}+\boldsymbol{b}_{1}(p+1)+\cdots+\boldsymbol{b}_{n-1}(p+1)^{n-1}}{\boldsymbol{a}_{0}+\boldsymbol{a}_{1}(p+1)+\cdots+\boldsymbol{a}_{n}(p+1)^{n}} \\
=\frac{\boldsymbol{B}_{0}+\boldsymbol{B}_{1} p+\cdots+\boldsymbol{B}_{n-1} p^{n-1}}{\boldsymbol{A}_{0}+\boldsymbol{A}_{1} p+\cdots+\boldsymbol{A}_{n} p^{n}}
\end{gathered}
$$

$G(p)$ given by (16) can be expanded through interval arithmetic (Appendix I) around $p=0$ as given in (17)-(18):

$$
\begin{gathered}
G(p)=\boldsymbol{\tau}_{0}+\tau_{0} \tau_{1} p+\boldsymbol{\tau}_{0} \tau_{1} \tau_{2} p^{2}+\cdots+\left(\prod_{i=0}^{n} \tau_{i}\right) p^{n}+\cdots \\
=\boldsymbol{t}_{0}+\boldsymbol{t}_{1} p+\boldsymbol{t}_{2} p^{2}+\cdots+\boldsymbol{t}_{n} p^{n}+\cdots
\end{gathered}
$$

where

$$
\boldsymbol{t}_{k}=\prod_{i=0}^{k} \tau_{i} \quad k=0,1,2, \cdots
$$

with 


$$
\tau_{k}=\left\{\begin{array}{cc}
\boldsymbol{B}_{0} / \boldsymbol{A}_{0} & k=0 \\
\boldsymbol{P}_{1}-\boldsymbol{Q}_{1} & k=1 \\
\left(\left(\boldsymbol{P}_{k}-\boldsymbol{Q}_{k}\right)-\sum_{i=1}^{k-1} \boldsymbol{Q}_{i}\left(\prod_{j=1}^{k-i} \tau_{j}\right)\right) \div\left(\prod_{l=1}^{k-1} \tau_{l}\right) & k \geq 2
\end{array}\right.
$$

where

and

$$
\boldsymbol{P}_{j}= \begin{cases}\frac{\boldsymbol{B}_{j}}{\boldsymbol{B}_{0}} & \forall j \in[0, n-1] \\ 0 & \forall j \geq n\end{cases}
$$

Putting $p=z-1$, (18) becomes

$$
\boldsymbol{Q}_{j}= \begin{cases}\frac{\boldsymbol{A}_{j}}{\boldsymbol{A}_{0}} & \forall j \in[0, n] \\ 0 & \forall j \geq n+1\end{cases}
$$

$$
G(z)=\boldsymbol{t}_{0}+\boldsymbol{t}_{1}(z-1)+\boldsymbol{t}_{2}(z-1)^{2}+\cdots+\boldsymbol{t}_{n}(z-1)^{n}+\cdots
$$

Therefore, the time moments of discrete HOIS (Shamash, 1974; Hwang and Shih, 1981) are

$$
\boldsymbol{T}_{i}= \begin{cases}\boldsymbol{t}_{i} & i=0 \\ (-1)^{i} \sum_{j=1}^{i} \frac{1}{j !}(\zeta)^{j} w_{i j}\left(\boldsymbol{t}_{j}\right) & i=1,2, \cdots\end{cases}
$$

where $\zeta$ is the sampling frequency and $w_{i j}$ is defined as

$$
w_{i j}= \begin{cases}1 & \text { for } j=1 \\ 0 & \text { for } i<j \\ 1 & \text { for } i=j \\ w_{i-1, j-1}+j w_{i-1, j} & \text { for } i>j\end{cases}
$$

Similarly, time moments of the ROIM are given by

$$
\hat{\boldsymbol{T}}_{i}= \begin{cases}\hat{\boldsymbol{t}}_{i} & i=0 \\ (-1)^{i} \sum_{j=1}^{i} \frac{1}{j !}(\zeta)^{j} w_{i j}\left(\hat{\boldsymbol{t}}_{j}\right) & i=1,2, \cdots\end{cases}
$$

where $\zeta$ is sampling frequency, $w_{i j}$ is given by (25) and $\hat{\boldsymbol{t}}_{i}$ for $i=0,1,2, \cdots$ have the same meaning as $\boldsymbol{t}_{i}$ for $i=0,1,2, \cdots$ in (19) for HOIS.

\subsection{Procedure to Obtain Denominator Coefficients of ROIM:}

In pole clustering method, cluster center is obtained by grouping of poles which is based on relative distance between the poles and desired order of ROIM in the process of modeling. In this process, each cluster is replaced by a single pole or a pair of complex conjugate poles. Separate clusters should be made for real poles and complex conjugate poles while all the poles on imaginary axis must be retained when obtaining ROIM through pole clustering method. The cluster center is obtained using IDM criterion (Vishwakarma and Prasad, 2008).

The IDM criterion is explained as follows:

The cluster center based on IDM criterion is given as

$$
\boldsymbol{C}^{c}=\left\{\left(\sum_{i=1}^{k}\left(\frac{1}{\boldsymbol{h}_{i}}\right)\right) \div k\right\}^{-1}
$$

where $\boldsymbol{C}^{c}$ is cluster center of $k$ real poles $\left(\boldsymbol{h}_{1}, \boldsymbol{h}_{2}, \cdots, \boldsymbol{h}_{k}\right)$ of the HOIS. 
The complex conjugate cluster centers in the form of $\boldsymbol{C}^{R} \pm j \boldsymbol{C}^{I}$ derived from $k$ pairs of complex conjugate poles $\left(\left(\boldsymbol{h}_{1}^{R} \pm j \boldsymbol{h}_{1}^{I}\right),\left(\boldsymbol{h}_{2}^{R} \pm j \boldsymbol{h}_{2}^{I}\right), \cdots,\left(\boldsymbol{h}_{k}^{R} \pm j \boldsymbol{h}_{k}^{I}\right)\right)$ are given as:

$$
\begin{aligned}
& \boldsymbol{C}^{R}=\left\{\left(\sum_{i=1}^{k}\left(\frac{1}{\boldsymbol{h}_{i}^{R}}\right)\right) \div k\right\}^{-1} \\
& \left.\boldsymbol{C}^{I}=\left\{\left(\sum_{i=1}^{k}\left(\frac{1}{\boldsymbol{h}_{i}^{I}}\right)\right) \div k\right\}^{-1}\right\}
\end{aligned}
$$

The denominator of ROIM can be obtained as:

Case 1: If all obtained cluster centers are real, the denominator of $r$ th-order model is given as

$$
D_{r}(z)=\prod_{i=1}^{r}\left(z-C_{i}^{c}\right)
$$

Case 2: If one pair of cluster center is complex conjugate and $(r-2)$ cluster centers are real, the denominator of $r$ th-order model is given as

$$
D_{r}(z)=\left(z-\left(C_{1}^{R}+j C_{1}^{I}\right)\right)\left(z-\left(C_{1}^{R}-j C_{1}^{I}\right)\right)\left(\prod_{i=3}^{r}\left(z-C_{i}^{c}\right)\right)
$$

Case 3: If all obtained cluster centers are complex conjugates, the denominator of $r$ th-order model is given as

$$
D_{r}(z)=\prod_{i=1}^{r / 2}\left(z-\left(C_{i}^{R}+j C_{i}^{I}\right)\right)\left(z-\left(C_{i}^{R}-j C_{i}^{I}\right)\right)
$$

\subsection{Procedure to Obtain Numerator Polynomial of ROIM:}

The time moments of the HOIS and ROIM are obtained by (24) and (26), respectively. The numerator polynomial of ROIM is obtained by minimizing error function which is weighted square sum of errors between first $r$ time moments of the HOIS to those of its ROIM, given by

$$
J_{C}=\sum_{i=1}^{r} \hat{\alpha}_{i}\left(\boldsymbol{T}_{i-1}-\hat{\boldsymbol{T}}_{i-1}\right)^{2}
$$

where $\hat{\alpha}_{i}$ are non-negative numbers.

Error function (32) is normalized as follows:

$$
J_{c}=\sum_{i=1}^{r} \alpha_{i}\left(1-\frac{\hat{\boldsymbol{T}}_{i-1}}{\boldsymbol{T}_{i-1}}\right)^{2}
$$

However, in (33), it is assumed that $\boldsymbol{T}_{i-1} \neq 0$. Using (26), (29), (30) and (31), (33) can solely be written in numerator parameters of ROIM as:

$$
J_{c}=\left(\hat{\boldsymbol{b}}_{0}, \hat{\boldsymbol{b}}_{1}, \cdots, \hat{\boldsymbol{b}}_{r-1}\right)
$$

\section{Luus-Jaakola Algorithm}

The error function (33) obtained is minimized using Luus-Jaakola (LJ) optimization technique (Luus and Jaakola, 1973). In Luus-Jaakola optimization algorithm, first initial test points over some region are chosen randomly after that region is contracted in every next iteration by a contraction factor while starting the iteration with the best estimate found in just previous iteration. There is more chance to get quick convergence using LJ optimization because the searches for the optimum values take place in the neighborhood of the best estimate found in the just previous iteration. Due to ease of programming and handling inequality constraints, algorithm has been applied in a variety of problems in optimal control (Singh et al., 2004; Singh et al., 2004).

Suppose error function to be minimized is

$$
J=f\left(x_{1}, x_{2}, \cdots, x_{N}\right)
$$

and the constraints to be satisfied are

$$
\begin{array}{lc}
c_{i}\left(x_{1}, x_{2}, \cdots, x_{N}\right) \geq 0 & i=1,2, \cdots, m \\
d_{j}\left(x_{1}, x_{2}, \cdots, x_{N}\right) \leq 0 & j=1,2, \cdots, n
\end{array}
$$

Following are the steps for L-J optimization:

Step. 1. Choose some reasonable initial value for $x_{i}$. Suppose it is $x_{i}^{0}, \quad i=1,2, \cdots, N$.

Choose reasonable initial region size for each variable. Suppose it is $\operatorname{reg}_{i}, \quad i=1,2, \cdots, N$. 
Step. 2. Take $R$ sets of random points to be used in each iteration. These are obtained as:

$$
x_{i}(j)=x_{i}^{0}(j)+\sigma \cdot \operatorname{reg}_{i}(j)
$$

where $\sigma=[-0.5,0.5], i=1,2, \cdots, N$ and $j=1,2, \cdots, R$.

Step. 3. Check the feasibility of constraints (36)-(37).

Step. 4. Evaluate the value of error function, for each feasible point.

Step. 5. Choose the minimum $J$ and corresponding $x_{i}, \quad i=1,2, \cdots N$.

Replace $x_{i}^{0}$ by this new $x_{i}$.

Step. 6. Reduce the region size $r e g(k)$ as

$$
\operatorname{reg}(k+1)=\gamma \cdot r e g(k)
$$

where $\gamma$ is a region contraction factor and $k$ is the iteration number.

Step. 7. Go to step 2 and repeat above procedure (step 2- 6) for $M$ iterations.

The suggested values of parameters are $R=100, \gamma=0.99$ and $M=600$.

\section{Numerical Section}

Consider the transfer function (Ismail et al., 1997) of a third-order discrete interval system

$$
G(z)=\frac{[1,2] z^{2}+[3,4] z+[8,10]}{[6,6] z^{3}+[9,9.5] z^{2}+[4.9,5] z+[0.8,0.85]}
$$

Suppose, a second-order model $(r=2)$ is desired given by the transfer function

$$
\left.\begin{array}{rl}
G_{2}^{p}(z) & =\frac{\left[\hat{b}_{0}^{-}, \hat{b}_{0}^{+}\right]+\left[\hat{b}_{1}^{-}, \hat{b}_{1}^{+}\right] z}{\left[\hat{a}_{0}^{-}, \hat{a}_{0}^{+}\right]+\left[\hat{a}_{1}^{-}, \hat{a}_{1}^{+}\right] z+\left[\hat{a}_{2}^{-}, \hat{a}_{2}^{+}\right] z^{2}} \\
& \equiv \frac{\hat{\boldsymbol{b}}_{0}+\hat{\boldsymbol{b}}_{1} z}{\hat{\boldsymbol{a}}_{0}+\hat{\boldsymbol{a}}_{1} z+\hat{\boldsymbol{a}}_{2} z^{2}} \equiv \frac{\hat{N}_{2}(z)}{\hat{D}_{2}(z)}
\end{array}\right\}
$$

The poles, calculated using (13), of the HOIS (40) are

$$
\left.\begin{array}{l}
\lambda_{1}^{F}=[-0.5340,-0.2680] \\
\lambda_{2}^{F}=[-0.7125,-0.5361] \\
\lambda_{3}^{F}=[-0.8534,-0.7203]
\end{array}\right\}
$$

Since poles (42) are real, thus, using (27) the cluster center obtained, by grouping $\lambda_{2}^{F}$ and $\lambda_{3}^{F}$, is

$$
C^{c}=[-0.7766,-0.6147]
$$

and retaining most dominant pole $\lambda_{1}^{F}$, denominator polynomial, using (29), is obtained as

$$
\left.\begin{array}{rl}
\hat{D}_{2}(z) & =\left(z-\lambda_{1}^{F}\right)\left(z-C^{c}\right) \\
& =(z-[-0.5340,-0.2680])(z-[-0.7766,-0.6147]) \\
& =[1,1] z^{2}+[0.8827,1.3106] z+[0.1647,0.4147] \\
& \equiv\left[\hat{a}_{2}^{-}, \hat{a}_{2}^{+}\right] z^{2}+\left[\hat{a}_{1}^{-}, \hat{a}_{1}^{+}\right] z+\left[\hat{a}_{0}^{-}, \hat{a}_{0}^{+}\right] \\
& \equiv \hat{\boldsymbol{a}}_{2} z^{2}+\hat{\boldsymbol{a}}_{1} z+\hat{\boldsymbol{a}}_{0}
\end{array}\right\}
$$

First two time moments of the HOIS (40), as given by (24), are

$$
\left.\begin{array}{l}
\boldsymbol{T}_{0}=[0.5621,0.7729] \\
\boldsymbol{T}_{1}=[0.6783,1.2520]
\end{array}\right\}
$$

and first two time moments, as given by (26), of the ROIM (41) are

$$
\left.\begin{array}{l}
\hat{\boldsymbol{T}}_{0}=\frac{\hat{\boldsymbol{b}}_{0}+\hat{\boldsymbol{b}}_{1}}{\hat{\boldsymbol{a}}_{0}+\hat{\boldsymbol{a}}_{1}+\hat{\boldsymbol{a}}_{2}} \\
\hat{\boldsymbol{T}}_{1}=-\left(\frac{\hat{\boldsymbol{b}}_{0}+\hat{\boldsymbol{b}}_{1}}{\hat{\boldsymbol{a}}_{0}+\hat{\boldsymbol{a}}_{1}+\hat{\boldsymbol{a}}_{2}}\right)\left(\frac{\hat{\boldsymbol{b}}_{1}}{\hat{\boldsymbol{b}}_{0}+\hat{\boldsymbol{b}}_{1}}-\frac{\hat{\boldsymbol{a}}_{1}+2 \hat{\boldsymbol{a}}_{2}}{\hat{\boldsymbol{a}}_{0}+\hat{\boldsymbol{a}}_{1}+\hat{\boldsymbol{a}}_{2}}\right)
\end{array}\right\}
$$


The Luss-Jaakola optimization algorithm is used to obtain optimal values of numerator parameters of ROIM for which (33) is minimized. Starting with various initial conditions and ranges, the algorithm converges to following optimal values

$$
\hat{\boldsymbol{b}}_{0}=[1.312,2.0934], \hat{\boldsymbol{b}}_{1}=[0.2369,0.5384]
$$

Thus, from (44) and (47), the second-order model becomes

$$
G_{2}^{p}(z)=\frac{[1.312,2.0934]+[0.2369,0.5384] z}{[0.1647,0.4147]+[0.8827,1.3106] z+[1,1] z^{2}}
$$

The second-order model presented in [19] is

$$
G_{2}^{o}(z)=\frac{[0.8845,0.9]+[0.5921,0.6055] z}{[0.1647,0.4147]+[0.8827,1.3106] z+[1,1] z^{2}}
$$

The step responses of some of the systems constructed with the help of Kharitonov polynomials (Appendix II) of numerator and denominator of HOIS (40), ROIM (49) in (Ismail et al., 1997) and proposed ROIM (48) are shown in Figure 1-2.



Figure 1. Step responses of HOIS and ROIMs.

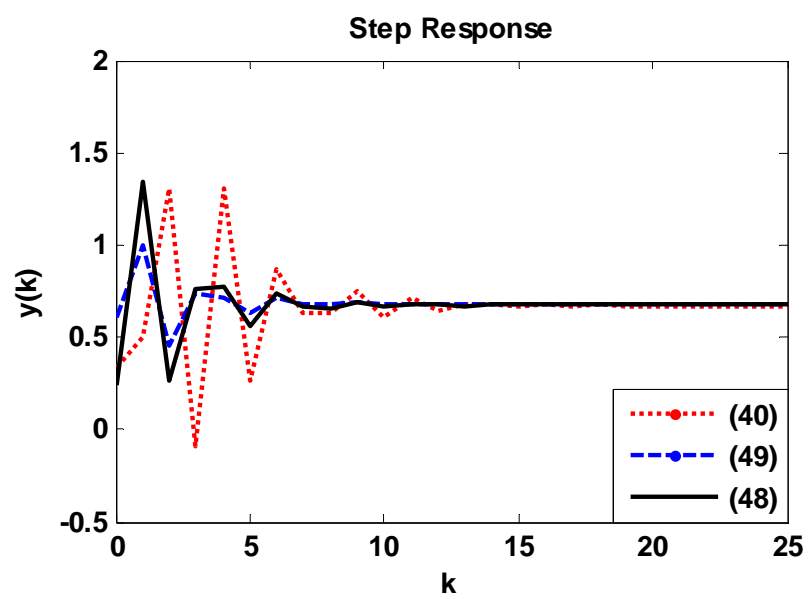

Figure 2. Step responses of HOIS and ROIMs.

It is clear from Figure 1 and 2 that the overall time response obtained by proposed method (48) is better than that of proposed in (Ismail et al., 1997). Hence, this confirms the applicability of proposed method to obtain ROIM for discrete HOISs.

\section{Conclusions}

A computer-aided method is proposed for obtaining ROIM of a given discrete HOIS in which the denominator of the ROIM is obtained by pole-clustering method and the numerator is obtained by minimizing an error function. The clustering of poles is done using IDM criterion and numerator parameters of ROIM are considered free in the process of minimization. The error function which is weighted squared sum of errors between first $r$ time moments of HOIS and those of ROIM is minimized using LuusJaakola optimization algorithm. It is worth mentioning here the methods based on combining neural network with pole clustering 
(Beyene, 2007). It would be interesting to compare the proposed method with this technique (Beyene, 2007) and this problem, in this context, is open to investigation.

\section{Appendix I}

INTERVAL ARITHMETIC

The rules of interval arithmetic (Sastry et al., 2000) are defined as follows:

Suppose, $\sigma \equiv\left[\sigma^{-}, \sigma^{+}\right]$and $\rho \equiv\left[\rho^{-}, \rho^{+}\right]$are two intervals.

Addition:

$$
\boldsymbol{\sigma}+\boldsymbol{\rho} \equiv\left[\sigma^{-}, \sigma^{+}\right]+\left[\rho^{-}, \rho^{+}\right]=\left[\sigma^{-}+\rho^{-}, \sigma^{+}+\rho^{+}\right]
$$

Subtraction:

$$
\sigma-\rho \equiv\left[\sigma^{-}, \sigma^{+}\right]+\left[\rho^{-}, \rho^{+}\right]=\left[\sigma^{-}-\rho^{+}, \sigma^{+}-\rho^{-}\right]
$$

Multiplication:

$$
\sigma . \rho \equiv\left[\sigma^{-}, \sigma^{+}\right] .\left[\rho^{-}, \rho^{+}\right]=\left[\min \left(\sigma^{-} \rho^{-}, \sigma^{-} \rho^{+}, \sigma^{+} \rho^{-}, \sigma^{+} \rho^{+}\right), \max \left(\sigma^{-} \rho^{-}, \sigma^{-} \rho^{+}, \sigma^{+} \rho^{-}, \sigma^{+} \rho^{+}\right)\right]
$$

Division:

$$
\boldsymbol{\sigma} / \boldsymbol{\rho} \equiv\left[\sigma^{-}, \sigma^{+}\right] /\left[\rho^{-}, \rho^{+}\right]=\left[\sigma^{-}, \sigma^{+}\right] /\left[1 / \rho^{+}, 1 / \rho^{-}\right] ; \quad \sigma / \sigma \equiv\left[\sigma^{-}, \sigma^{+}\right] /\left[\sigma^{-}, \sigma^{+}\right]=1
$$

\section{Appendix II}

\section{KHARITONOV POLYNOMIALS}

Consider a family $f$ of real interval polynomials (Kharitonov, 1978):

$$
\begin{aligned}
A(z) & =\left[\sigma_{0}^{-}, \sigma_{0}^{+}\right]+\left[\sigma_{1}^{-}, \sigma_{1}^{+}\right] z+\left[\sigma_{2}^{-}, \sigma_{2}^{+}\right] z^{2}+\cdots+\left[\sigma_{n}^{-}, \sigma_{n}^{+}\right] z^{n} \\
& \equiv \sigma_{0}+\sigma_{1} z+\sigma_{2} z^{2}+\cdots+\sigma_{n-1} z^{n-1}+\sigma_{n} z^{n}
\end{aligned}
$$

The four Kharitonov polynomials associated with $A(z)$ are given below:

$$
\begin{aligned}
& A_{f}^{1}(z)=\sigma_{0}^{-}+\sigma_{1}^{-} z+\sigma_{2}^{+} z^{2}+\sigma_{3}^{+} z^{3}+\sigma_{4}^{-} z^{4}+\cdots \\
& A_{f}^{2}(z)=\sigma_{0}^{+}+\sigma_{1}^{-} z+\sigma_{2}^{-} z^{2}+\sigma_{3}^{+} z^{3}+\sigma_{4}^{+} z^{4}+\cdots \\
& A_{f}^{3}(z)=\sigma_{0}^{+}+\sigma_{1}^{+} z+\sigma_{2}^{-} z^{2}+\sigma_{3}^{-} z^{3}+\sigma_{4}^{+} z^{4}+\cdots \\
& A_{f}^{4}(z)=\sigma_{0}^{-}+\sigma_{1}^{+} z+\sigma_{2}^{+} z^{2}+\sigma_{3}^{-} z^{3}+\sigma_{4}^{-} z^{4}+\cdots
\end{aligned}
$$

\section{References}

Aoki M., 1968. Control of large-scale dynamic systems by aggregation, IEEE Trans. Auto. Cont., Vol. 13, pp. 246-253.

Baker G.A. and Graves-Morris P.R., 1981. Padé approximants, Part-II: Extensions and Applications, London: Addison-Wesley.

Baker G.A., 1975. Essenstials of Padé approximants, New York: Academic.

Bandyopadhyay B., Ismail O. and Gorez R., 1994. Routh-Padé approximation for interval systems, IEEE Trans. Auto. Cont., Vol. 39, No. 12, pp. 2454-2456.

Bandyopadhyay B., Upadhye A. and Ismail O., 1997. $\gamma-\delta$ Routh approximation for interval systems, IEEE Trans. on Automatic Control, Vol. 42, No. 8, pp. 1127-1130.

Beyene W.T., 2007. Low-order rational approximation of interconnects using neural-network based pole-clustering techniques, IEEE International Symposium on Circuits and Systems (ISCAS 2007), pp. 1501-1504.

Dolgin Y. and Zeheb E., 2003. On Routh-Padé model reduction of interval systems, IEEE Trans. on Auto. Control, Vol. 48, No. 9, pp. 1610-1612.

Glover K., 1984. All optimal Hankel-norm approximants of linear multivariable systems and their $H^{\infty}$ error bounds, Int. Jr. Control, Vol 39, No. 6, pp. 1115-1193.

Hutton M.F. and Friedland B., 1975. Routh approximations for reducing the order of linear time-invariant systems, IEEE Trans. Autom. Control, Vol 20, pp. 329-337.

Hwang C. and Shih Y.P., 1981. On the time moments of discrete systems, Int. J. Contr., Vol. 34, pp. 1227-1228.

Ismail O. and Bandyopadhyay B., 1995. Model reduction of linear interval systems using Padé approximation, IEEE International symposium on Circuits and systems (ISCAS), Vol.2, pp. 1400-1403.

Ismail O., Bandyopadhyay B. and Gorez R., 1997. Discrete interval system reduction using Padé approximation to allow retention of dominant poles, IEEE Trans. on Circuits and Systems_I: Fundamental theory and applications, Vol. 44, No. 11, pp.10751078.

Kharitonov V.L., 1978. Asymptotic stability of an equilibrium position of a family of system of linear differential equation, 
Differential'Nye Uravenia, Vol. 14, pp. 1483-1485.

Luus R. and Jaakola T.H.I., 1973. Direct search and systematic reduction of size of search region, AIChE J., Vol. 19, pp. 760-766.

Prajapati N.L., Chandra D. and Seshachalam D., 2007. Corrections and Comments to "Model reduction of discrete linear systems via frequency-domain balanced structure, IEEE Trans. on Circuits and Systems I, Vol. 54, No. 3, pp. 682-683.

Rao A.S., Lamba S.S. and Rao S.V., 1978. Routh-approximant time-domain reduced-order modelling for single-input single-output systems, IEE Proc., Control Theory Appl., Vol. 125, pp. 1059-1063.

Sastry G.V.K.R., Raja G. and P M. Rao, 2000. Large scale interval system modeling using Routh approximants, IET Journal, Vol. 36, No. 8, pp. 768-769.

Shamash Y., 1974. Continued fraction methods for the reduction of discrete-time dynamic systems, Int. J. Contr., Vol. 20, pp. 267275.

Shamash Y., 1974. Stable reduced order models using Padé type approximation, IEEE Trans. Auto. Cont., Vol. 19, pp. 615-616.

Shamash Y., 1975. Model reduction using the Routh stability criterion and the Padé approximation technique, Int. J. Control, Vol. 21, pp. 475-484.

Singh V., Chandra D. and Kar H., 2004. Improved Routh-Padé approximants: a computer-aided approach, IEEE Transactions on Automatic Control, Vol. 49, No. 2, pp. 292-296.

Singh V., Chandra D. and Kar H., 2004. Optimal Routh approximants through integral squared error minimisation: computer-aided approach, IEE Proc.-Control Theory and Appl., Vol. 151, No. 1, pp. 53-58.

Singh V.P. and Chandra D., 2011. Model reduction of discrete interval system using dominant poles retention and direct series expansion method, IEEE International Power Engineering and Optimization Conference (PEOCO-2011), pp. 27-30.

Sinha N.K. and Kuszta B., 1983. Modeling and identification of dynamic systems, New York: Van Nostand Reinhold, pp. 133-163.

Vishwakarma C.B. and Prasad R., 2008. Clustering method for reducing order of linear system using Pade approximation, IETE Journal of Research, Vol. 54, No. 5, pp. 326-330.

\section{Biographical notes}

Vinay Pratap Singh was born in Etawah, U.P., India on July 01, 1987. He graduated from the Uttar Pradesh Technical University, Lucknow, India with B.Tech degree in Electrical Engineering in 2007 and completed his M.Tech. in Control \& Instrumentation at EED, MNNIT, Allahabad in July 2009. He is currently pursuing Ph.D. in Electrical Engineering Department at MNNIT, Allahabad. His research interests include Model reduction and Design of controllers.

D. Chandra received the Bachelor's degree in electrical engineering from Madan Mohan Malaviya Engineering College, Gorakhpur, India, in 1973, and the Master's and Ph.D. degrees in electrical engineering from the Motilal Nehru National Institute of Technology Allahabad, India, in 1979, and 2002, respectively. Since the past 35 years, he has been with Motilal Nehru National Institute of Technology, where he is currently a Professor. His research interests include control systems, reduced order modeling, optimization, and converters' control.

Received January 2012

Accepted February 2012

Final acceptance in revised form March 2012 